\title{
Pulsating stars in open clusters
}

\author{
T. Arentoft ${ }^{1}$, L.M. Freyhammer ${ }^{2,3}$, M.Y. Bouzid ${ }^{2}$, C. Sterken ${ }^{2}$ and S. Frandsen ${ }^{1}$ \\ ${ }^{1}$ Aarhus University, Ny Munkegade, Bldg. 520, DK-8000 Aarhus C, Denmark \\ ${ }^{2}$ Vrije Universiteit Brussel, Pleinlaan 2, B-1050 Brussels, Belgium \\ ${ }^{3}$ Royal Observatory of Belgium, Ringlaan 3, B-1180 Uccle, Belgium
}

\begin{abstract}
We report studies that are carried out with the aim of searching for and studying short-period variable stars in northern open clusters. The background for these studies is described along with the results that have been obtained up to now.
\end{abstract}

\section{Introduction}

The overall goal of studying short-period pulsating stars, such as $\delta$ Scuti or $\beta$ Cep stars, is to do asteroseismology - i.e. to use the pulsational frequencies to constrain stellar models. Such constraints become even tighter if parameters like age and distance can be determined independently, as is the case in clusters. Furthermore, studying pulsating stars in clusters using CCD photometry has the advantage that several variables can be observed simultaneously, making the observations very efficient. Large numbers of pulsating stars can be found in globular clusters (see e.g. Bono et al. 2003), but the photometric precision is limited by crowding and therefore, in general, only high-amplitude variables are studied. In open clusters, on the other hand, the number of variables is much smaller, but the fields are typically only semi-crowded although still containing a significant number of constant stars, making high-precision (mmag) photometry possible. In such systems, low-amplitude, multi-periodic variables like $\delta$ Scuti or $\beta$ Cep stars can be studied to high precision.

Several very interesting open clusters are known. An excellent example is NGC 6231 (e.g. Arentoft et al. 2001, Sterken et al. 2002). This cluster contains, apart from a set of eclipsing binaries, 7-8 $\beta$ Cep stars, 3 SPB candidates, $4 \delta$ Scuti stars and $3 \gamma$ Dor candidates as well as several variables of presently unknown type. This rich variety of variables makes NGC 6231 one of the most attractive clusters to study as it ultimately will offer the possibility for comparative studies of variables of different types formed under the same conditions. Further, extensive studies are underway on this cluster, as it is the main subject of the $\mathrm{PhD}$ studies of M.Y. Bouzid. Among other interesting clusters are NGC 6134 (Frandsen et al. 1996) which contains 6-7 $\delta$ Scuti stars or NGC 3293 (Balona \& Engelbrecht 1983) and NGC 4755 (Stankov et al. 2002) which both contain a number of $\beta$ Cep stars.

However, these clusters are positioned in the southern skies while most small (1-2 m) telescopes are situated in the northern hemisphere. Such telescopes are the optimal instruments for obtaining cluster time series because of their typical field of view (FoV) (several arcmin) and availability for long, uninterrupted time series. For this reason, a part of the work related to STACC (an observing network focusing on studying cluster variables, Frandsen (1992)) has in recent years been to search for northern open clusters with a population $(>5)$ of pulsating stars within a small FoV (a few arcmin). The strategy for this project along with the results that have been obtained so far are summarized in the two following Sections. 


\section{Observing strategy}

The search has mainly focused on finding multi-periodic $\delta$ Scuti stars in the open clusters. To do so, target clusters were carefully selected in order, obviously, to make the search efficient. The selection criteria are mainly the cluster distance (1-2 kpc is preferred) and age (0.3-1.0 Gyr). The former ensures relatively compact, semi-crowded fields with many stars but few crowding problems as well as the possibility to do spectroscopy with medium sized telescopes, the latter that many stars are in the $\delta$ Scuti instability strip. This is because the cluster turn-off from the main-sequence for these clusters will be located in the $\delta$ Scuti instability region of the HR-diagram. A number of clusters that fits this description are compiled by Frandsen \& Arentoft (1998a), who also discuss three steps in the observations of $\delta$ Scuti stars in open clusters: step I is locating interesting targets by obtaining a few (3-4) nights of high-quality time-series to identify pulsating stars, in step II target information is collected (membership information, physical parameters from spectroscopy and photometry). Step III is a coordinated multisite campaign using telescopes around the globe in order to secure continuous monitoring and abundant data (500+ hours of photometry). Then follows the confrontation with the models (asteroseismology). Below we describe the progress on two clusters - NGC 7062 and NGC 1817 - for both of which step II has been reached.

\section{Results on NGC 7062 and NGC 1817}

NGC 7062 was observed with the 2.54-m Nordic Optical Telescope in August 2000. Four nights had been allocated to the project, but only two were clear while the remaining two nights had poor conditions. The data reduction was carried out with the programme MOMF (Kjeldsen \& Frandsen 1992) which combines PSF and aperture photometry and therefore is very robust towards variable observing conditions. Despite the mixed weather conditions, sufficient high-quality data was obtained to allow $>4 \sigma$ detections of pulsational frequencies with amplitudes as low as $1.2 \mathrm{mmag}$. From these observations, Freyhammer et al. (2001) detected 15 variable stars within a FoV of only $6.5 \times 6.5$ arcmin. Of these variables, at least 13 are $\delta$ Scuti stars and of those, 10 were found to pulsate in two or more frequencies and are thus multiperiodic.

Another cluster that has been studied is NGC 1817. This cluster has a relatively large extent in the sky of almost half a degree and only a part of the cluster has been searched for pulsating stars so far. However, the part that has been investigated has turned out to be extremely interesting. First, Frandsen \& Arentoft (1998b) found 7 potential $\delta$ Scuti stars from two nights of observations with the IAC $80-\mathrm{cm}$ telescope at Tenerife, Spain. This was then followed up by new observations in December 2002, again with the Nordic Optical Telescope. The observations were collected during 5 nights and included both time-series observations in $B$ and $V$ and $u v b y$ standard data. Preliminary results from the analysis of these observations were presented by Arentoft et al. (2004) where the detection of 14 new variable stars was announced. This brings the total number of known variable stars in NGC 1817 to 19 , of which 12 are multi-periodic $\delta$ Scuti stars. An example of a newly detected $\delta$ Scuti star is shown in Fig. 1. Furthermore, Balaguer-Núnez et al. (1998) carried out a proper motion study of NGC 1817. Based on their results (and for some stars that are not included in their study, on the position in the HR-diagram) 11 of the $12 \delta$ Scuti stars are probable cluster members.

These findings make both NGC 7062 and NGC 1817 prime targets for further investigations within the STACC network. It is therefore time now to stop searching for target clusters and instead to complete step II and start planning step III for these two clusters. Indeed, we are contemplating to launch a multi-site campaign on NGC 1817 in one of the coming observing seasons, probably in the northern winter 2004-2005. 


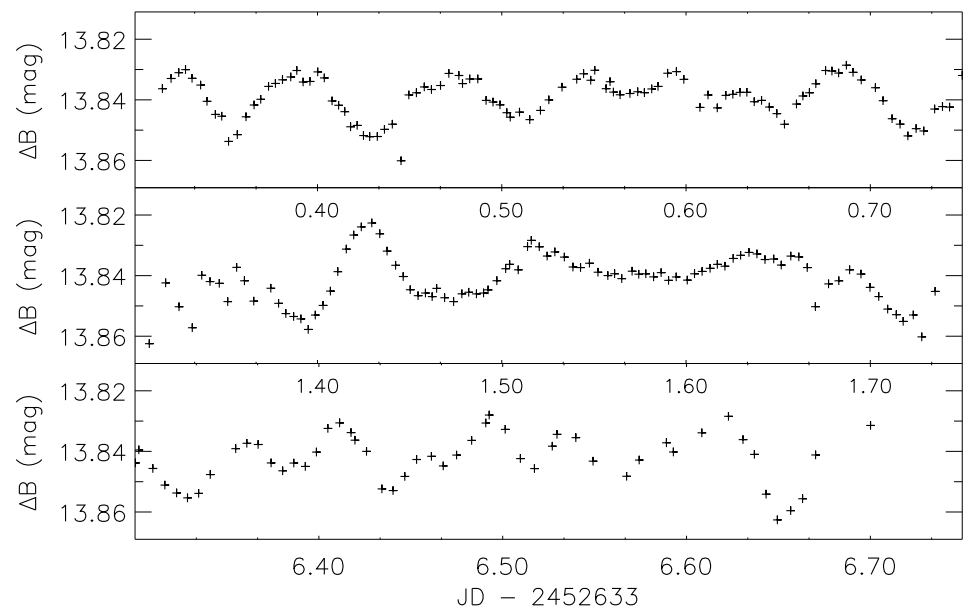

Figure 1: A new multiperiodic $\delta$ Scuti star detected with the new NGC 1817 data. The data are less abundant during the last night because alternating $B$ and $V$ images were obtained.

Acknowledgments. TA acknowledges financial support from The Danish Natural Science Research Council, partly through the project "Stellar structure and evolution - new challenges from ground and space observations" carried out at Aarhus University and Copenhagen University. LMF and CS acknowledge support from the project IUAP P5/36 financed by the Belgian State, Federal Office for Scientific, Technical and Cultural Affairs. MYB and CS acknowledge financial support from the Belgian Fund for Scientific Research (FWO).

\section{References}

Arentoft, T., Freyhammer, L.M., Bouzid, M.Y., Sterken, C., Frandsen, S. 2004, to appear in "Variable Stars in the Local Group “, ASP Conf. Ser., D.W. Kurtz \& K. Pollard, eds.

Arentoft, T., Sterken, C., Knudsen, M.R. et al. 2001, A\&A 380, 599

Balaguer-Núnez, L., Tian, K.P., Zhao, J.L. 1998, AAS 133, 387

Balona, L.A., Engelbrecht, C.A. 1983, MNRAS 202, 293

Bono, G., Petroni, S., Marconi, M. 2003, ASP. Conf. Ser. 292, "Interplay of Periodic, Cyclic and

Stochastic Variability in Selected Areas of the H-R Diagram ", ed. C. Sterken, 71

Frandsen, S. 1992, Delta Scuti Star Newsletter (Univ. of Vienna) 5, 12

Frandsen, S., Balona, L.A., Viskum, M. et al. 1996, A\&A 308, 132

Frandsen, S., Arentoft, T. 1998a, J. Astron. Data 4, 6

Frandsen, S., Arentoft, T. 1998b, A\&A 333, 524

Freyhammer, L.M., Arentoft, T., Sterken, C. 2001, A\&A 368, 580

Kjeldsen, H., Frandsen, S. 1992, PASP 104, 413

Stankov, A., Handler, G., Hempel, M., Mittermayer, P. 2002, MNRAS 336, 189

Sterken, C., Knudsen, M.R., Duerbeck, H.W. et al. 2002, ASP Conf. Ser. Vol. 256, "Observational

Aspects of Pulsating B- and A Stars ", eds. C. Sterken and D.W. Kurtz, 183 